\section{Serum Interleukin 18 as a Diagnostic Remission Criterion in Systemic Juvenile Idiopathic Arthritis}

\section{To the Editor:}

With the development of new therapeutic agents and combination treatment strategies, more children with systemic juvenile idiopathic arthritis (sJIA) can experience protracted periods of low disease activity levels and, in some cases, complete disease quiescence. These advances create a need for the development of validated criteria that precisely describe the clinical state of disease quiescence.

We previously reported that serum interleukin 18 (IL-18) levels in patients with sJIA were extremely high during the active phase and remained significantly elevated even when other markers of disease activity normalized ${ }^{1}$. We also reported that serum IL-18 levels at birth in a healthy infant born to a woman with active adult-onset Still's disease were markedly increased, and this increase persisted for about 1 month $^{2}$. These findings indicate that it takes several months for extremely elevated serum IL-18 levels to normalize under physiological conditions.

In our study, we serially measured serum IL-18 levels in 11 patients with sJIA (age $10.2 \pm 7.6 \mathrm{yrs}$, male:female $=3: 8$ ) until they had relapsed or achieved remission to investigate the kinetics of serum IL-18 levels from the active phase to remission. Further, we investigated the correlation between serum IL-18 and IL-6 levels in these patients. Among 11 patients, 4 patients were enrolled in our previous study ${ }^{1}$, while 7 patients were enrolled in our other study ${ }^{3}$. The criteria defining the active phase of sJIA were active arthritis, fever, rash, hepatosplenomegaly, generalized lymphadenopathy, and serositis, as well as increased erythrocyte sedimentation rate (ESR) and C-reactive protein (CRP) levels. The criteria for inactive phase of sJIA were as follows: no active arthritis, no fever; no rash, serositis, splenomegaly, or generalized lymphadenopathy; normal ESR or CRP level, and a physician's global assessment of disease activity indicating clinical disease quiescence ${ }^{4,5}$. The criteria for remission are as follows: remission while receiving medication, a minimum of 6 continuous months of inactive disease while receiving medication, clinical remission while not receiving medication, or 12 months of inactive disease while not receiving any medication ${ }^{4,5}$. Serum IL-18 and IL-6 levels were determined using a commercial ELISA as we previously reported ${ }^{1}$. The limit of detection of IL-18 and IL-6 in our assay were $<12.5 \mathrm{pg} / \mathrm{ml}$ and $<3.0$ $\mathrm{pg} / \mathrm{ml}$. In our assay, serum IL-18 and IL-6 levels of 28 healthy controls (age $8.8 \pm 7.3 \mathrm{yrs})$ were $140.5(76-255) \mathrm{pg} / \mathrm{ml}$ and $<3.0 \mathrm{pg} / \mathrm{ml}^{1}$, respectively. The protocol of our study was approved by the Institutional Review Board of Kanazawa University, and all the patients provided informed consent. All patients were treated with high-dose steroid, including methylprednisolone pulse therapy ( $30 \mathrm{mg} / \mathrm{kg} /$ day, 3 days). In addition to steroid, 6 patients were treated with cyclosporine and 2 patients were treated with tocilizumab (TCZ; $8 \mathrm{mg} / \mathrm{kg}$, every 2 weeks). Followup periods were at least over 15 months ( 15 mos-7 yrs). As we previously reported, serum IL-6 levels in patients receiving TCZ therapy are higher compared to those in patients not receiving TCZ therapy because IL- 6 receptor-mediated consumption of IL-6 is inhibited by the unavailability of TCZ-free IL-6 receptor ${ }^{6,7}$. Therefore, the correlation between serum IL-18 and IL- 6 levels was determined in 9 patients not receiving TCZ therapy.

Of the 11 patients, 5 had no relapse (group A); $3 / 5$ patients achieved remission while not taking medication. Remission while not taking medication was maintained for over 3 years. Two patients achieved remission while taking medication. The other 6 patients experienced relapse during withdrawal of steroid within 12 months after disease onset (group B). Of the 6 patients, 4 experienced relapse during the inactive phase and the other 2 patients experienced relapse during remission while taking medication. As shown in Figure 1A, the longitudinal examination of group A patients showed that serum IL-18 levels decreased to the levels $<1000$ $\mathrm{pg} / \mathrm{ml}$ in inactive phase and normalized in remission phase. In contrast, longitudinal examination of group B patients clearly demonstrated a sustained elevation of serum IL-18 levels $(>1000 \mathrm{pg} / \mathrm{ml}$ ) during the inactive phase (Figure 1B). The serum IL-18 levels correlated positively with the serum IL-6 levels ( $p<0.0001$; Figure 1C). However, serum IL-18 levels remained elevated even in inactive phase after serum IL-6 levels normalized.

Wallace, et al proposed remission criteria for JIA, which acknowledged that patients with JIA may be in 1 of 2 states, either active or inactive disease $\mathrm{s}^{4,5}$. The following are included in the criteria for inactive disease: no active arthritis, no fever; no rash, serositis, splenomegaly, or generalized lymphadenopathy; normal erythrocyte sedimentation rate or C-reactive protein level, and a physician's global assessment of disease activity indicating clinical disease quiescence. Inactive disease can be further divided into clinical remission while taking medication (a minimum of 6 continuous mos of inactive disease while receiving medication) and clinical remission while not taking medication (12 mos of inactive disease while not receiving any medications).

In our study, serum IL-18 levels in the remission group (group A) were elevated during the inactive phase, but these significantly decreased to normal levels during the remission phase. These findings support the accuracy of the Wallace criteria for clinical remission. Further, serum IL-18 levels may be useful as a diagnostic laboratory criterion for clinical remission in SJIA.

In the clinical setting of sJIA, relapses of acute sJIA flares have occurred during the inactive phase ${ }^{1}$. In our study, 6 patients experienced relapses during the inactive phase or early phase of remission while taking medication. Longitudinal examination in these patients demonstrated sustained elevation of serum IL-18 levels during these phases. These findings indicate that persistent inflammation associated with macrophage activation cannot be controlled. Serum IL-18 levels may reflect the biological activity of the immune system and disease activity of sJIA.

The limitation of our study is the small number of patients with sJIA who were evaluated. It would be necessary to perform larger scale studies to confirm our preliminary data and draw firm conclusions. Despite this limitation, our results indicate that serum IL-18 levels reflect the biological activities of the immune system in sJIA and may predict the prognosis of sJIA. Serum IL-18 levels may be useful as a diagnostic laboratory criterion for clinical remission in sJIA.

MASAKI SHIMIZU, MD, PHD, Department of Pediatrics, School of Medicine, Institute of Medical, Pharmaceutical and Health Sciences, Kanazawa University, Kanazawa; YASUO NAKAGISHI, MD, Department of Pediatric Rheumatology, Hyogo Prefectural Kobe Children's Hospital, Hyogo; AKIRA YOSHIDA, MD, PhD, Department of Pediatrics, Japanese Red Cross Wakayama Medical Center, Wakayama; AKIHIRO YACHIE, MD, PhD, Department of Pediatrics, School of Medicine, Institute of Medical, Pharmaceutical and Health Sciences, Kanazawa University, Kanazawa, Japan.

Address correspondence to Dr. M. Shimizu, Department of Pediatrics, School of Medicine, Institute of Medical, Pharmaceutical, and Health Sciences, Kanazawa University, 13-1 Takaramachi, Kanazawa 920-8641, Japan.E-mail: shimizum@staff.kanazawa-u.ac.jp

\section{REFERENCES}

1. Shimizu M, Yokoyama T, Yamada K, Kaneda H, Wada H, Wada T, et al. Distinct cytokine profiles of systemic-onset juvenile idiopathic arthritis-associated macrophage activation syndrome with particular emphasis on the role of interleukin-18 in its pathogenesis. Rheumatology 2010;49:1645-53.

2. Shimizu M, Sakakibara Y, Kawano M, Yachie A. Transient impairment of NK cell function in an infant born to a mother with adult-onset Still's disease: perinatal effect of maternal IL-18. Clin Immunol 2012;143:273-4.

3. Shimizu M, Nakagishi Y, Yachie A. Distinct subsets of patients with systemic juvenile idiopathic arthritis based on their cytokine profiles. Cytokine 2013;61:345-8.

4. Wallace CA, Ruperto N, Giannini E; Childhood Arthritis and Rheumatology Research Alliance; Pediatric Rheumatology 
$\mathrm{IL}-18$

$(\mathrm{pg} / \mathrm{ml})$

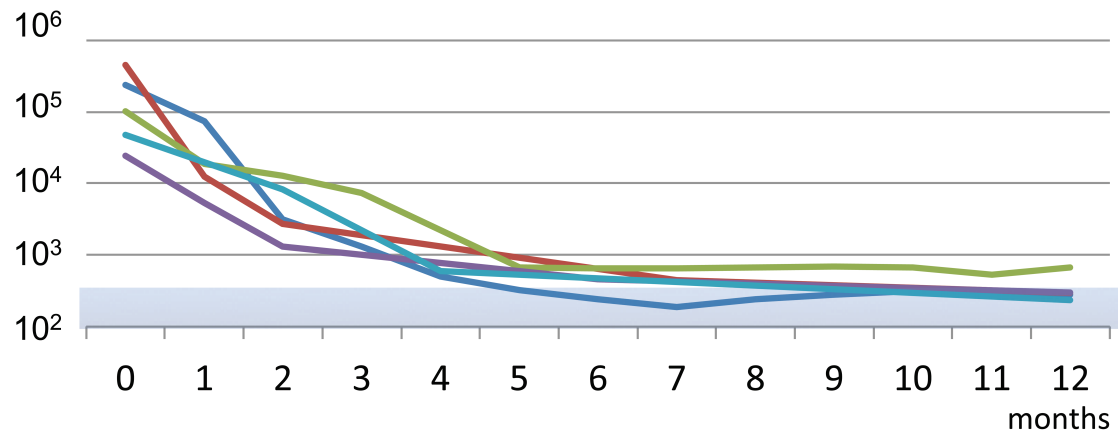

B

$\mathrm{IL}-18$

$(\mathrm{pg} / \mathrm{ml})$

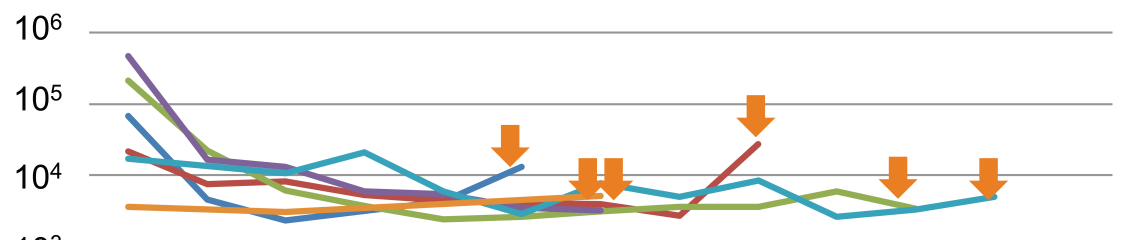

$10^{3}$

$10^{2}$
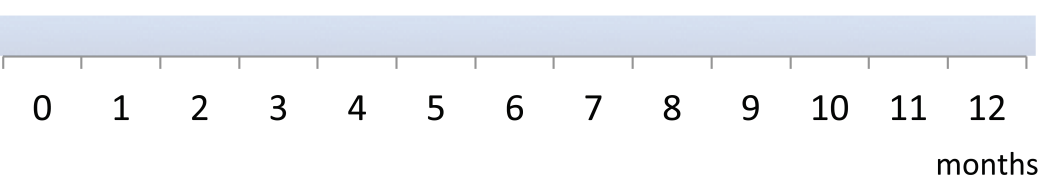

C

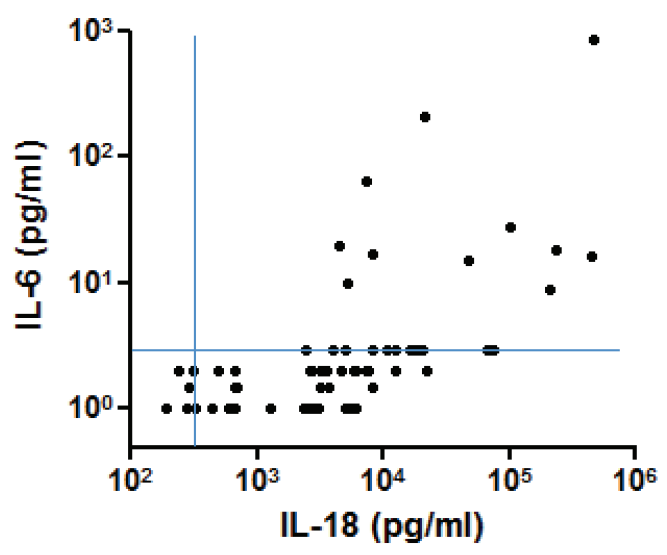

Figure 1. Longitudinal examination of serum interleukin 18 (IL-18) levels in patients with systemic juvenile idiopathic arthritis. A. Group A: patients with remission. B. Group B: patients with relapse. Orange arrows show the timing of the relapses in each patient. C. Correlation between serum IL-18 and IL-6 levels. Serum IL-6 levels are within normal limits whereas IL-18 levels remain elevated.

International Trials Organization; Pediatric Rheumatology Collaborative Study Group. Preliminary criteria for clinical remission for select categories of juvenile idiopathic arthritis. J Rheumatol 2004;31:2290-4.
5. Wallace CA, Giannini EH, Huang B, Itert L, Ruperto N; Childhood Arthritis Rheumatology Research Alliance; Pediatric Rheumatology Collaborative Study Group; Paediatric Rheumatology International Trials Organisation. American College 
of Rheumatology provisional criteria for defining clinical inactive disease in select categories of juvenile idiopathic arthritis. Arthritis Care Res 2011;6:929-36.

6. Shimizu M, Nakagishi Y, Kasai K, Yamasaki Y, Miyoshi M, Takei $\mathrm{S}$, et al. Tocilizumab masks the clinical symptoms of systemic juvenile idiopathic arthritis-associated macrophage activation syndrome: the diagnostic significance of interleukin-18 and interleukin-6. Cytokine 2012;58:287-94.

7. Nishimoto N, Terao K, Mima T, Nakahara H, Takagi N, Kakehi T. Mechanisms and pathologic significances in increase in serum interleukin-6 (IL-6) and soluble IL-6 receptor after administration of an anti-IL-6 receptor antibody, tocilizumab, in patients with rheumatoid arthritis and Castleman disease. Blood 2008; 112:3959-64.

J Rheumatol 2014;41:11; doi:10.3899/jrheum.140025 